\title{
Lung metastases in metastatic gastric cancer: pattern of lung metastases and clinical outcome
}

\author{
Jee Hyun Kong $\cdot$ Jeeyun Lee $\cdot$ Chin-A Yi $\cdot$ \\ Se Hoon Park · Joon Oh Park · Young Suk Park • \\ Ho Yeong Lim $\cdot$ Keon Woo Park $\cdot$ Won Ki Kang
}

Received: 10 May 2011 / Accepted: 23 September 2011/Published online: 29 October 2011

(C) The International Gastric Cancer Association and The Japanese Gastric Cancer Association 2011

\begin{abstract}
Background There are only limited data regarding pulmonary metastasis from gastric cancer. Therefore, we analyzed large series of gastric cancer with pulmonary metastasis and analyzed their clinical characteristics and treatment outcome to enhance perception of metastatic gastric cancer.

Methods Of 20,187 advanced gastric cancer patients treated between 1995 and 2007, 193 (0.96\%) were identified to have pulmonary metastasis from gastric cancer. The pulmonary lesions were detected at chest computed tomography (CT) scan or plain chest X-ray and/or abdominal pelvic CT scan covering the lower part of the lungs, and were divided into three patterns: lymphangitic, hematogenous, and pleural.

Results The most frequently observed pattern of lung metastasis was hematogenous metastasis $(52.3 \%)$ followed
\end{abstract}

J. H. Kong - J. Lee $\cdot$ S. H. Park - J. O. Park ·

Y. S. Park · H. Y. Lim · W. K. Kang (ه)

Division of Hematology-Oncology, Department of Medicine,

Sungkyunkwan University, Samsung Medical Center,

Seoul 135-710, Korea

e-mail: wkkang@skku.edu

\section{J. H. Kong}

Division of Hematology-Oncology, Department of Medicine, Wonju Christian Hospital, College of Medicine, Yonsei

University, Wonju, Kangwon-Do, Korea

C.-A. Yi

Department of Radiology, Samsung Medical Center,

Sungkyunkwan University School of Medicine, Seoul, Korea

K. W. Park

Department of Hematology-Oncology,

Dankook University School of Medicine, Cheonan, Korea by pleural (35.2\%) and lymphangitic (26.4\%). Patients who had hematogenous pulmonary metastasis were significantly associated with hepatic metastasis $(p=0.004)$ and male sex $(p=0.012)$. Patients with lymphangitic metastasis were significantly associated with concomitant bone $(p=0.010)$ and bone marrow $(p=0.029)$ metastasis. In case of pleural metastasis, it was positively correlated with gastrectomy history $(p=0.015)$ and the presence of peritoneal metastasis $(p=0.020)$. After a median follow-up duration of 87 (9-162) months, the median survival after diagnosis of pulmonary metastasis was 4 (0-67) months. Conclusion The most frequently observed pattern of lung metastasis was hematogenous metastasis $(52.3 \%)$ followed by pleural $(35.2 \%)$ and lymphangitic $(26.4 \%)$ in gastric cancer patients. Among gastric cancer patients with lung metastases, patients with pleural metastasis or lymphangitic metastasis had shorter survival with 1.5-2-fold increased risk of deaths.

Keywords Pulmonary metastasis - Gastric cancer . Hematogenous metastasis - Lymphangitic metastasis · Pleural metastasis

\section{Introduction}

Despite the increasing early detection rate owing to widespread screening with endoscopy, especially in Asian countries, long-term survival of gastric cancer patients is still low [1-3]. Five-year survival for unresectable, metastatic gastric cancer is only around 5-20\%, with median overall survival being less than 1 year [4], and it is one of the leading cause of cancer deaths worldwide [5, 6]. In order to understand tumor biology and to optimize treatment outcome, it is essential to understand patterns of 
spread for specific cancer types. In our previous study, we demonstrated that the presence of bone metastasis or clinically significant ascites was a significantly poor prognostic factor for survival in metastatic gastric cancer patients receiving palliative first-line chemotherapy [7].

The pulmonary parenchyma is a common site for metastatic deposits of malignant neoplasm because the lung is highly vascularized with ample lymphatic drainage [8]. Malignant neoplasm often metastasized to the lung via hematogenous spread, resulting in variable-sized lesions from small nodules to extremely large masses, or via lymphatics, generating streaky or linear infiltrative lesions in the lung [8]. In gastric cancer patients, their major feature of recurrence is intra-abdominal spread [9], and the incidence of pulmonary metastasis is relatively rare [10, 11]. Moreover, the prognosis of extra-abdominal tumor spread becomes obvious [9], and the clinical features and treatment outcome of pulmonary metastasis in gastric cancer have not been extensively studied. Therefore, we systematically reviewed the tumor registry to retrieve gastric cancer patients with pulmonary metastases and analyzed the treatment outcome and prognostic factors in this subgroup of patients.

\section{Method}

\section{Patients}

From 1995 to 2007, we reviewed the tumor registry at Samsung Medical Center to retrieve data on gastric cancer patients who were diagnosed with pulmonary metastases from gastric cancer. The registry includes all gastric cancer patients with early to advanced or metastatic gastric cancer. The eligibility criteria for case selection were as follows: (1) pathologic or radiologic diagnosis of pulmonary metastasis from gastric adenocarcinoma, (2) imaging studies including chest computed tomography (CT) scan, plain chest X-ray, and/or abdominal pelvic CT available for review, and (3) presence of pulmonary visceral metastases (not limited to thoracic lymph nodes). We systematically reviewed the medical records and collected the following clinical data: demographic data, surgical record, pathologic features, treatment modalities, concomitant metastatic sites other than the lung, and vital status.

Patterns of pulmonary metastasis

Pulmonary metastases were categorized as follows according to imaging studies (Fig. 1):

1. Hematogenous metastasis: multiple variable-sized lung nodules in CT and/or chest X-ray (limited hematogenous metastasis: $\leq 3$ metastatic lung nodules).
2. Pleural metastasis: pleural thickening and/or pleural effusion identified in CT scan and/or at plain chest $\mathrm{X}$-ray without definite concomitant disease that can produce the pleural effusion, such as pneumonia or heart failure.

3. Lymphangitic metastasis: interlobar, septal, or fissural markings and/or irregular contour of bronchovascular markings in CT and/or chest X-ray.

\section{Statistical analysis}

Mann-Whitney test was used for numerical comparison between two groups. Fisher's exact test and chi-square test were applied for categorical variables, and one-way ANOVA was for continuous variables. Pearson's method was used for evaluation of correlation. Overall survival (OS) was estimated using the Kaplan-Meier product-limit method. OS was measured from the date of diagnosis of pulmonary metastasis to the date of death or the last follow-up visit. Survival rates were compared for statistical differences by using log-rank analysis. A prognostic model was established by searching all variables that significantly influenced OS at a level of $p$ values less than or equal to 0.05 in the univariate analysis. Multivariate analysis was performed using stepwise Cox proportional hazards regression modeling. $p$ values less than 0.05 were considered statistically significant, and all $p$ values correspond to two-sided significance tests.

\section{Results}

\section{Patient characteristics}

Of the 20,187 gastric cancer patients reviewed, 193 $(0.96 \%)$ were identified as having lung metastasis. Their clinical characteristics are summarized in Table 1 . The median age was 53 (27-74) years, and male gender (63.7\%) was predominant. Both chest X-ray and chest CT images were available in $117(60.6 \%)$ patients, and 76 patients had serial chest X-rays available for review. For gastric cancer with pulmonary metastases, Bormann type III was the most commonly observed type, and about two thirds of these patients developed lung metastasis in the later course of the disease. At the time of diagnosis of pulmonary metastases, the lung was the solitary metastatic organ in $40(20.7 \%)$ patients, while 153 patients had concomitant metastatic sites other than the lung, such as peritoneal seeding $(42.5 \%)$, liver $(40.9 \%)$, bone $(17.6 \%)$, or bone marrow $(5.7 \%)$. Approximately $30 \%$ of patients did not received chemotherapy, and most patients received 

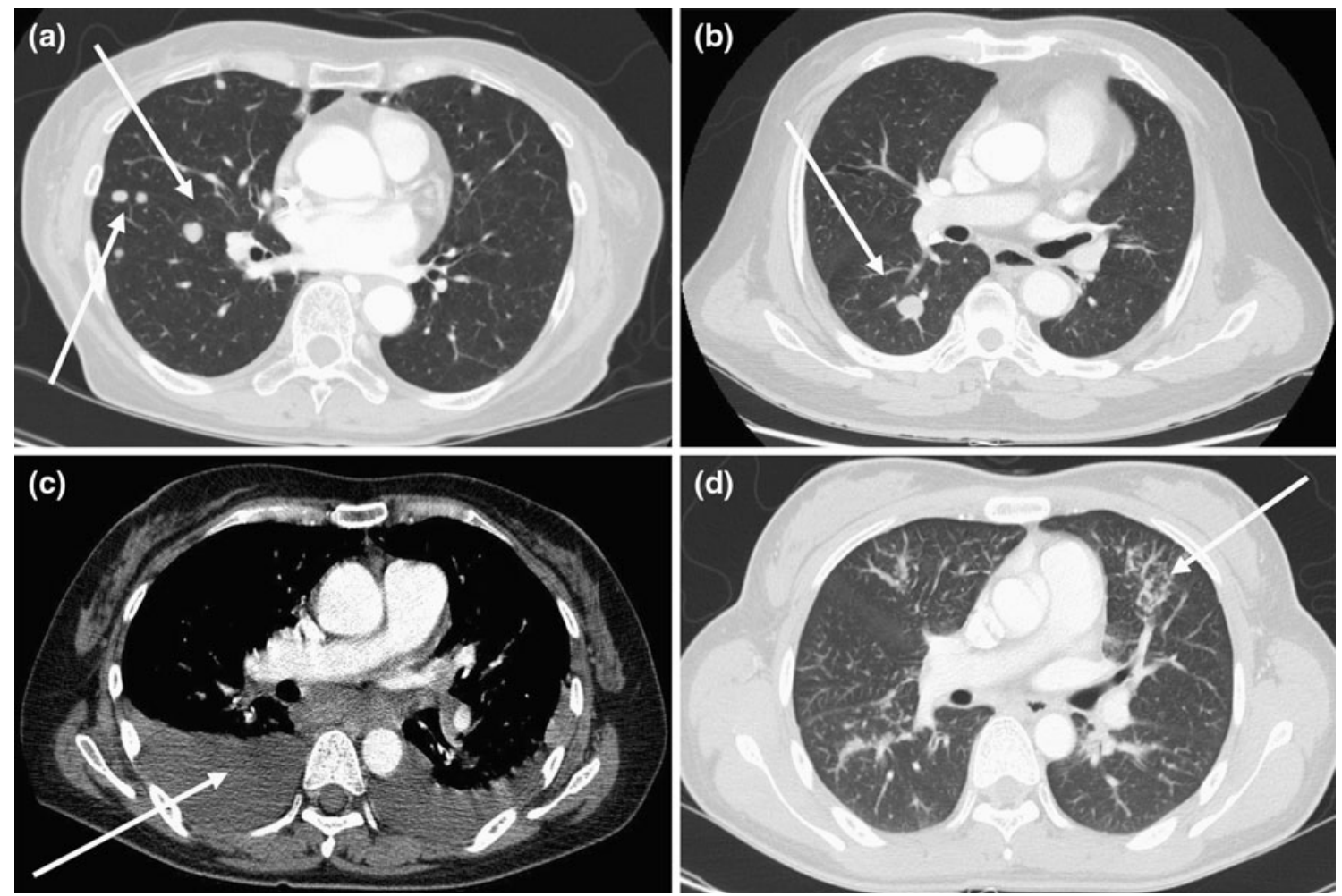

Fig. 1 Chest CT scans of each pulmonary metastasis pattern in gastric cancer. a Hematogenous metastasis: multiple nodules were observed in the right lower lobe (arrows). b Limited hematogenous metastasis: one nodule was observed in the right lower lobe (arrow).

conventional 5-FU-based or taxane-based chemotherapy. One patient underwent metastasectomy for a solitary lung nodule.

\section{Patterns of lung metastasis}

Of 193 patients, 101 (52.3\%) patients were categorized as having hematogenous metastases, $68(35.2 \%)$ as having pleural metastases, and $51(26.4 \%)$ as having lymphangitic metastatic lesions (Table 2; Fig. 1). Among them, 24 $(12.6 \%)$ patients had a mixed pattern of pulmonary metastasis as described in Fig. 2. To compare clinical characteristics according to the pulmonary metastatic patterns, we divided patients into four groups according to their pulmonary metastatic patterns based on imaging studies: hematogenous, lymphangitic, pleural, and mixed (Table 2). For the lymphangitic group, the mean age was younger $(p<0.001)$, a higher portion were of male sex $(p=0.027)$, and there was a higher likelihood for concomitant liver metastases $(55.7 \%)$ or peritoneal seeding $(54.1 \%)$. Pleural metastases were associated with diffuse type according to the Lauren classification when compared to other groups, although this was not statistically significant $(p=0.081)$. In case of pleural metastasis, it was c Pleural effusion: both sides of the pleural effusion were observed without lung parenchymal lesion. d Lymphangitic metastasis. Diffuse septal thickening with ground-glass opacity was noted, especially on the left upper lobe (arrow)

positively correlated with gastrectomy history $(p=0.015)$ and the presence of peritoneal metastasis $(p=0.020)$.

\section{Survival}

The median follow-up duration was 87.0 (9-162) months. The median time from diagnosis to lung metastasis was 6 (0-138) months, which was not different according to the patterns of pulmonary metastasis $(p=0.164)$. The median survival after diagnosis of pulmonary metastasis was 4.0 (0-67) months (Fig. 3). Exceptionally, there was one patient who developed a single lung metastasis 23 months after curative total gastrectomy for well-differentiated gastric adenocarcinoma. The patient underwent lung metastasectomy and has been disease free for 37 months since surgery. In univariate analysis, advanced stage at the time of diagnosis $(p=0.004)$, absence of gastrectomy history $(p=0.045)$, presence of ascites $(p=0.024)$ or bone metastasis $(p=0.009)$, and lymphangitic $(p=0.002)$ or pleural metastasis $(p=0.000)$ demonstrated poor survival with statistical significance, but other variables, including sex, diagnosis time of pulmonary metastasis, concomitant metastasis to liver, bone marrow and palliative chemotherapy, did not predict survival (data not shown). As 
Table 1 Patients characteristics

\begin{tabular}{|c|c|c|}
\hline & $n$ & $\begin{array}{l}\text { Total } \\
(\%, n=193)\end{array}$ \\
\hline Median age (years) & $53(27-74)$ & - \\
\hline Sex male/female & $123 / 70$ & $63.7 / 36.3$ \\
\hline Gastrectomy history & 112 & 58 \\
\hline \multicolumn{3}{|l|}{ Tumor location } \\
\hline Upper third & 39 & 20.2 \\
\hline Middle third & 67 & 34.7 \\
\hline Lower third & 64 & 33.2 \\
\hline Unknown & 23 & 11.9 \\
\hline \multicolumn{3}{|l|}{ Bormann type } \\
\hline I & 3 & 1.6 \\
\hline II & 29 & 15.0 \\
\hline III & 101 & 52.3 \\
\hline IV & 27 & 14.0 \\
\hline EGC & 7 & 3.6 \\
\hline Unknown & 26 & 13.5 \\
\hline \multicolumn{3}{|l|}{ Pathology } \\
\hline \multicolumn{3}{|l|}{ Histological type } \\
\hline $\begin{array}{l}\text { Adenocarcinoma, } \\
\text { well differentiated }\end{array}$ & 8 & 4.1 \\
\hline $\begin{array}{l}\text { Adenocarcinoma, moderate } \\
\text { differentiated }\end{array}$ & 46 & 23.8 \\
\hline $\begin{array}{l}\text { Adenocarcinoma, poorly } \\
\text { differentiated }\end{array}$ & 84 & 43.5 \\
\hline Signet ring cell & 32 & 16.6 \\
\hline Others & 23 & 11.9 \\
\hline \multicolumn{3}{|l|}{ Lauren classification } \\
\hline Intestinal & 28 & 14.5 \\
\hline Diffuse & 55 & 28.5 \\
\hline Mixed & 2 & 1.0 \\
\hline Unknown & 108 & 56.0 \\
\hline \multicolumn{3}{|l|}{ Venous invasion } \\
\hline Presence & 18 & 9.6 \\
\hline Absence & 12 & 6.2 \\
\hline Unknown & 163 & 84.5 \\
\hline \multicolumn{3}{|l|}{ Lymphatic invasion } \\
\hline Presence & 66 & 34.2 \\
\hline Absence & 7 & 3.6 \\
\hline Unknown & 120 & 62.2 \\
\hline \multicolumn{3}{|l|}{ Perineural invasion } \\
\hline Presence & 36 & 18.7 \\
\hline Absence & 15 & 7.8 \\
\hline Unknown & 142 & 73.3 \\
\hline \multicolumn{3}{|l|}{ Diagnosis time of pulmonary metastasis } \\
\hline $\begin{array}{l}\text { At the time of diagnosis of gastric } \\
\text { cancer }\end{array}$ & 66 & 34.2 \\
\hline After diagnosis of gastric cancer & 127 & 65.8 \\
\hline \multicolumn{3}{|l|}{ Metastatic organ } \\
\hline Pulmonary metastases alone & 40 & 20.7 \\
\hline Concomitant metastasis & 153 & 79.3 \\
\hline
\end{tabular}

Table 1 continued

\begin{tabular}{lcc}
\hline & $n$ & $\begin{array}{l}\text { Total } \\
(\%, n=193)\end{array}$ \\
\hline Peritoneal seeding & 82 & 42.5 \\
Liver & 79 & 40.9 \\
Bone & 34 & 17.6 \\
Bone marrow & 11 & 5.7 \\
Chemotherapy after pulmonary metastasis & \\
None & 58 & 30.0 \\
Fluorouracil based & 80 & 41.5 \\
Taxane based & 49 & 25.4 \\
Others & 6 & 3.1 \\
\hline
\end{tabular}

Table 2 Frequency of pulmonary metastasis patterns in gastric cancer patients

\begin{tabular}{lc}
\hline Metastasis pattern & Frequency $(\%)$ \\
\hline Hematogenous pattern & $101(52.3)$ \\
Pleural pattern & $68(35.2)$ \\
Lymphangitic pattern & $51(26.4)$ \\
\hline
\end{tabular}

shown in Table 3, the pattern of lung metastases was associated with significantly different clinical features in sexual distribution $(p=0.0152), \quad$ Bormann type $(p=0.0107)$, histologic types $(p=0.0064)$, Lauren classification $(p=0.0436)$, lymphatic invasion $(p=0.0091)$, and metastatic organ sites $(p \leq 0.0001)$. For metastatic pattern, hematogenous lung metastasis was significantly associated with liver metastasis when compared with lymphangitic metastasis $(p=0.0006)$. In contrast, pleural metastases were considerably associated with peritoneal seeding rather than other visceral metastases with statistical significance ( $p=0.0024)$ by Fisher's exact test using the permutation method and Bonferroni's correction for multiple testing. At multivariate analysis, lymphangitic $(\mathrm{HR}=1.861,95 \%$ CI 1.164-2.977, $p=0.009)$ and pleural metastasis $(\mathrm{HR}=2.213,95 \%$ CI 1.419-3.450, $p=0.000$ ) were significant predictable parameters for poor survival.

\section{Discussion}

Depending on the cancer origin and cell subtype, metastasis may emerge quickly in multiple organs or in particular organs after a protracted latent period [12]. In an attempt to enhance understanding of the clinical impact of lung metastasis from gastric cancer, we analyzed the clinical features and treatment outcome in 193 gastric cancer patients with lung metastasis. To the best of our 
Fig. 2 The percentage of pulmonary metastasis patterns in gastric cancer patients

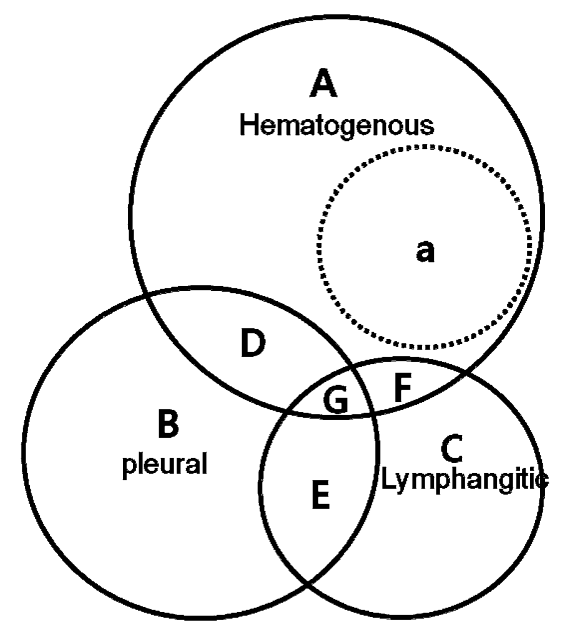

A. Hematogenous metastasis $n=86(44.5 \%)$ (a. limited $\mathrm{n}=30(15.5 \%)$ )

B. Pleural metastasis $\mathrm{n}=47(24.4 \%)$

C. Lymphangitic metastasis $n=36(18.7 \%)$

D. Hematogenous and pleural metastasis $n=9(4.7 \%)$

E. Lymphangitic and pleural metastasis $n=9(4.7 \%)$

F. Hematolymphangitic metastasis $n=3(1.6 \%)$

G. Hematolymphangitic and pleural metastasis $n=3(1.6 \%)$ knowledge, this represents the largest study focusing on gastric cancer with lung metastasis.

Koga et al. [10] reported 7 (0.5\%) pulmonary metastases in 1,314 gastric cancer patients and Kanemitsu et al. [11] 22 $(0.7 \%)$ in 3,076 patients. In concordance with previous studies, the incidence of lung metastasis from gastric cancer was $0.96 \%(193 / 20,187)$ in our series. Of 193 patients, 40 $(20.7 \%)$ had pulmonary metastasis as the only organ site of metastasis. The most frequently observed pattern of lung metastasis was hematogenous $(52.3 \%)$ followed by pleural (35.2\%) and lymphangitic metastasis (26.4\%) (Table 2; Fig. 1). Approximately $80 \%$ of patients with lung metastasis had concomitant metastasis to other organs with the peritoneum $(57.0 \%)$ being the most common site followed by liver $(40.9 \%)$, bone (17.6\%), and bone marrow $(5.7 \%)$. Hematogenous lung metastasis was significantly associated with liver metastasis when compared with lymphangitic metastasis $(p=0.0006)$. In contrast, pleural metastases were considerably associated with peritoneal seeding rather than other visceral metastases with statistical significance $(p=0.0024)$. Hence, there is a high likelihood that gastric cancer patients with hematogeneous lung metastasis will carry concomitant liver metastasis. Moreover, gastric cancer with lymphangitic metastasis was significantly associated with bone $(p=0.0312)$ and bone marrow $(p=0.0258)$ metastasis rather than other visceral metastases such as liver when compared with hematogenous metastases by Fisher's exact test. Hence, the underlying mechanism for metastasis governing lymphangitic spread and hematogeneous spread may be distinct in gastric cancer.

Lymphangitic $\quad(\mathrm{HR}=1.861,95 \%$ CI $1.164-2.977$, $p=0.009)$ lung metastasis was a significant poor prognostic factor for survival at multivariate analysis. Likewise, pleural metastasis was associated with concomitant peritoneal metastasis $(p=0.020)$ and was a significant predictor for poor survival with HR of 2.213 (95\% CI 1.419-3.450,

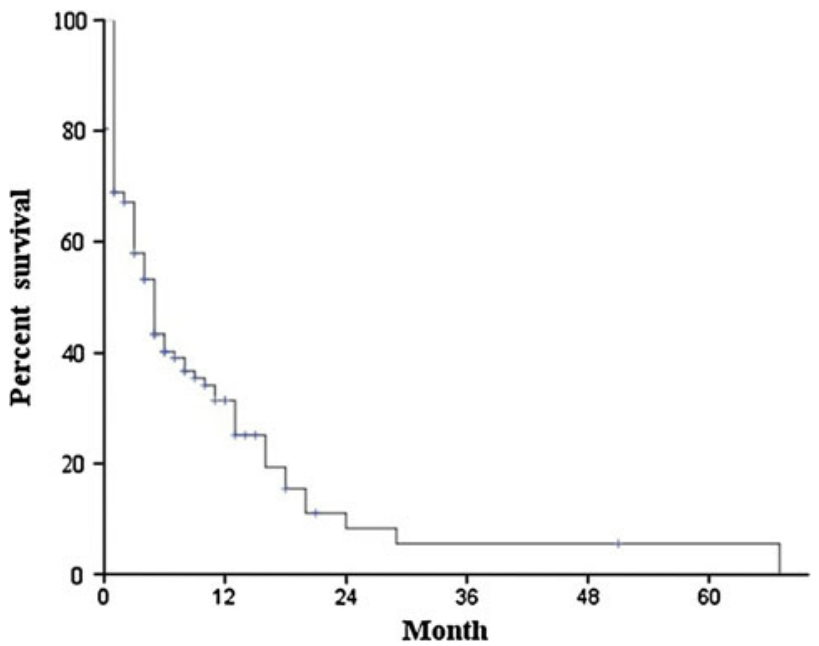

Fig. 3 Survival curve using Kaplan-Meier method. The median overall survival after pulmonary metastasis in advanced gastric cancer was 4 (0-67) months

$p=0.000)$. In support of this, only 21 (10.9\%) patients had an overlapping pattern of lung metastasis as described in Fig. 2, which suggests commitment lung metastasis and spread in lymphangitic, hematogenous, or pleural areas at an early time of dissemination. Therefore, the exploration of predictive factors for lymphangitic spread and pleural spread is warranted to improve survival in metastatic gastric cancer patients.

Conspicuously, one gastric cancer patient with solitary lung metastasis who underwent lung metastasectomy survived without recurrence of disease for 37 months at the time of this writing. The potential benefit of metastasectomy of lung nodules has not been actively pursued in metastatic gastric cancer, yet because of the incidence of pulmonary metastasis alone, especially surgically resectable solitary metastasis after resection of gastric cancer is very low [11]. However, there were several case reports of long-term survival after resection of pulmonary metastases 
Table 3 Patients' clinical characteristics according to pattern of lung metastases

\begin{tabular}{|c|c|c|c|c|c|}
\hline & $\begin{array}{l}\text { Hematogenous } \\
(n=36)\end{array}$ & $\begin{array}{l}\text { Pleural } \\
(n=47)\end{array}$ & $\begin{array}{l}\text { Lymphangitic } \\
(n=86)\end{array}$ & $\begin{array}{l}\text { Mixed } \\
(n=24)\end{array}$ & $p$ value* \\
\hline Age & $46.1( \pm 11.4)$ & $51.1( \pm 11.8)$ & $56.3( \pm 10.5)$ & $49.0( \pm 12.0)$ & $\mathrm{NC}$ \\
\hline \multicolumn{6}{|l|}{ Sex } \\
\hline M & $16(44.4 \%)$ & $29(61.7 \%)$ & $58(67.4 \%)$ & $20(83.3 \%)$ & \multirow[t]{2}{*}{0.0152} \\
\hline $\mathrm{F}$ & $20(55.6 \%)$ & $18(38.3 \%)$ & $28(32.6 \%)$ & $4(16.7 \%)$ & \\
\hline \multicolumn{6}{|l|}{ Tumor location } \\
\hline Upper third & $9(25.5 \%)$ & $12(25.5 \%)$ & $14(16.3 \%)$ & $4(16.7 \%)$ & \multirow[t]{4}{*}{0.2493} \\
\hline Middle third & $17(47.2 \%)$ & $14(29.8 \%)$ & $29(33.7 \%)$ & $7(29.2 \%)$ & \\
\hline Lower third & $9(31.9 \%)$ & $15(31.9 \%)$ & $33(38.4 \%)$ & $7(29.2 \%)$ & \\
\hline Unknown & $1(2.7 \%)$ & $6(12.8 \%)$ & $10(11.6 \%)$ & $6(11.6 \%)$ & \\
\hline \multicolumn{6}{|l|}{ Bormann type } \\
\hline I & $0(0.0 \%)$ & $0(0.0 \%)$ & $3(3.5 \%)$ & $0(0.0 \%)$ & \multirow[t]{5}{*}{0.0107} \\
\hline II & $2(5.6 \%)$ & $5(10.6 \%)$ & $18(20.9 \%)$ & $4(16.7 \%)$ & \\
\hline III & $17(47.2 \%)$ & $24(51.1 \%)$ & $49(57.0 \%)$ & $11(45.8 \%)$ & \\
\hline IV & $9(25.0 \%)$ & $11(23.4 \%)$ & $3(3.5 \%)$ & $4(16.7 \%)$ & \\
\hline Unknown & $8(22.2 \%)$ & $7(14.9 \%)$ & $13(15.1 \%)$ & $5(20.8 \%)$ & \\
\hline \multicolumn{6}{|l|}{ Histological type } \\
\hline AD well & $1(2.7 \%)$ & $1(2.1 \%)$ & $6(7.0 \%)$ & $0(0.0 \%)$ & \multirow[t]{5}{*}{0.0064} \\
\hline AD moderate & $7(19.4 \%)$ & $7(14.9 \%)$ & $30(34.9 \%)$ & $2(8.3 \%)$ & \\
\hline $\mathrm{AD}$ poor & $18(50.0 \%)$ & $24(51.1 \%)$ & $31(36.0 \%)$ & $11(45.8 \%)$ & \\
\hline Signet ring cell & $8(22.2 \%)$ & $11(23.4 \%)$ & $7(8.1 \%)$ & $6(25.0 \%)$ & \\
\hline Others & $2(5.6 \%)$ & $1(2.1 \%)$ & $12(14.0 \%)$ & $1(4.2 \%)$ & \\
\hline \multicolumn{6}{|l|}{ Lauren classification $(n=85)$} \\
\hline Intestinal & $5(13.9 \%)$ & $5(10.6 \%)$ & $17(20.0 \%)$ & $1(4.2 \%)$ & \multirow[t]{4}{*}{0.0436} \\
\hline Diffuse & $7(19.4 \%)$ & $22(46.8 \%)$ & $18(20.9 \%)$ & $8(33.3 \%)$ & \\
\hline Mixed & $1(2.7 \%)$ & $0(0.0 \%)$ & $1(1.2 \%)$ & $0(0.0 \%)$ & \\
\hline Unknown & $23(63.9 \%)$ & $20(42.6 \%)$ & $50(58.1 \%)$ & $15(58.1 \%)$ & \\
\hline \multicolumn{6}{|l|}{ Lymphatic invasion $(n=73)$} \\
\hline Absence & $0(0.0 \%)$ & $0(0.0 \%)$ & $7(8.1 \%)$ & $0(0.0 \%)$ & \multirow[t]{3}{*}{0.0091} \\
\hline Presence & $12(33.3 \%)$ & $25(53.2 \%)$ & $23(26.7 \%)$ & $6(26.7 \%)$ & \\
\hline Unknown & $24(66.7 \%)$ & $22(46.8 \%)$ & $56(65.1 \%)$ & $18(65.1 \%)$ & \\
\hline \multicolumn{6}{|c|}{ Metastatic organ (may not add up to 193 due to multiple sites checked) } \\
\hline Liver $(n=79)$ & $9(25.0 \%)$ & $15(31.9 \%)$ & $44(51.1 \%)$ & $11(45.8 \%)$ & \multirow[t]{4}{*}{$<0.0001$} \\
\hline Bone $(n=34)$ & $12(33.3 \%)$ & $12(25.5 \%)$ & $5(5.8 \%)$ & $5(20.8 \%)$ & \\
\hline Peritoneal seeding $(n=82)$ & $23(63.9 \%)$ & $15(31.9 \%)$ & $60(69.8 \%)$ & $13(54.2 \%)$ & \\
\hline Bone marrow $(n=11)$ & $3(8.3 \%)$ & $4(8.5 \%)$ & $1(1.2 \%)$ & $3(12.5 \%)$ & \\
\hline
\end{tabular}

* Fisher's exact test using MCMC

from gastric cancer $[13,14]$, and there might be a role for lung metastasectomy in gastric cancer as observed in colorectal cancer. One of the challenges for conducting a clinical study in such a population would be a limited number of patients as we observed only nine cases with isolated oligonodular lung metastasis. Complicating the issue, the possibility of second primary lung cancer should be considered since $0.5-1.7 \%$ of gastric cancer patients develop lung cancer.
In conclusion, we found that only $20 \%$ of gastric cancer patients with lung metastasis have isolated pulmonary metastasis. The most frequently observed pattern of lung metastasis was hematogenous metastasis (52.3\%) followed by pleural $(35.2 \%)$ and lymphangitic (26.4\%) metastasis in gastric cancer. Importantly, patients with pleural metastasis or lymphangitic metastasis had shorter survival with 1.5-2-fold increased risk of deaths. More aggressive treatment needs to be sought 
in this subgroup of patients with a rapidly deteriorating clinical course.

\section{References}

1. Inokuchi K. Prolonged survival of stomach cancer patients after extensive surgery and adjuvant treatment: an overview of the Japanese experience. Semin Surg Oncol. 1991;7:333-8.

2. Kim JP, Kwon OJ, Oh ST, Yang HK. Results of surgery on 6589 gastric cancer patients and immunochemosurgery as the best treatment of advanced gastric cancer. Ann Surg 1992;216:269-78 (discussion 78-9).

3. Siewert JR, Bottcher K, Roder JD, Busch R, Hermanek P, Meyer HJ. Prognostic relevance of systematic lymph node dissection in gastric carcinoma. German Gastric Carcinoma Study Group. Br J Surg. 1993;80:1015-8.

4. Kamangar F, Dores GM, Anderson WF. Patterns of cancer incidence, mortality, and prevalence across five continents: defining priorities to reduce cancer disparities in different geographic regions of the world. J Clin Oncol. 2006;24:2137-50.

5. Wu CW, Hsieh MC, Lo SS, Lui WY, P'Eng FK. Results of curative gastrectomy for carcinoma of the distal third of the stomach. J Am Coll Surg. 1996;183:201-7.

6. Wanebo HJ, Kennedy BJ, Chmiel J, Steele G Jr, Winchester D, Osteen R. Cancer of the stomach. A patient care study by the American College of Surgeons. Ann Surg. 1993;218:583-92.
7. Lee J, Lim T, Uhm JE, Park KW, Park SH, Lee SC, et al. Prognostic model to predict survival following first-line chemotherapy in patients with metastatic gastric adenocarcinoma. Ann Oncol. 2007;18:886-91.

8. Fred A. Mettler. Essentials of radiology. Amsterdam: Elsevier; 2005.

9. Yoo CH, Noh SH, Shin DW, Choi SH, Min JS. Recurrence following curative resection for gastric carcinoma. Br J Surg. 2000;87:236-42.

10. Koga S, Takebayashi M, Kaibara N, Nishidoi H, Kimura O, Kawasumi H, et al. Pathological characteristics of gastric cancer that develop hematogenous recurrence, with special reference to the site of recurrence. J Surg Oncol. 1987;36:239-42.

11. Kanemitsu Y, Kondo H, Katai H, Nakayama H, Asamura H, Tsuchiya R, et al. Surgical resection of pulmonary metastases from gastric cancer. J Surg Oncol. 1998;69:147-50.

12. Nguyen DX, Bos PD, Massague J. Metastasis: from dissemination to organ-specific colonization. Nat Rev Cancer. 2009;9:274-84.

13. Nakayama H, Ichinose S, Kato Y, Ito H, Masui K, Kameda Y. Long-term survival after a surgical resection of pulmonary metastases from gastric cancer: report of a case. Surg Today. 2008;38:150-3.

14. Nakahashi C, Kinoshita T, Konishi M, Nakagohri T, Inoue K, Oda $\mathrm{T}$, et al. Long-term survival achieved by repeated resections of metachronous pulmonary and adrenal metastases of alphafetoprotein-producing gastric cancer: report of a case. Surg Today. 2004;34:784-7. 